\title{
Antidepressant Prescriptions, Including Tricyclics, Continue to Increase in Canadian Children
}

\author{
Aysha Lukmanji, BA\&Sc, ${ }^{1}$ Tamara Pringsheim, MD, ${ }^{2,3}$ Andrew G. Bulloch, PhD, ${ }^{1,3}$ David G. Stewart, MA, \\ Parco Chan, MSc, ${ }^{4}$ Ali Tehrani, BA, ${ }^{4}$ and Scott B. Patten, MD, PhD ${ }^{1,3,5}$
}

\begin{abstract}
Objective: Few studies have longitudinally followed trends in antidepressant prescribing for Canadian children following the Black Box warning issued in 2004. Using a national data source, we aim to describe trends in antidepressant recommendations for Canadian children ages 1-18 during 2012 to 2016.

Methods: A database called the Canadian Disease and Therapeutic Index (CDTI), provided by IQVIA, was used to conduct analyses. The CDTI dataset collects a quarterly sample of pediatric antidepressant recommendations, projected using a weight procedure from a dynamic sample of 652 Canadian office-based physicians. The term "recommendations" is used because nonprescription drugs may be recommended and there is no confirmation in the database that the prescriptions were filled or medications taken. The data were collected from 2012 to 2016 and the sample population was projected by IQVIA to be representative of the entire Canadian pediatric population.

Results: The total number of projected antidepressant recommendations for children increased from 2012 to 2016. Selective serotonin reuptake inhibitors were the most recommended class of antidepressants. Analysis indicated that fluoxetine was the most frequently recommended drug. Findings also suggest that recommendations for tricyclic antidepressants (TCAs) are increasing, but predominantly for reasons other than treatment of depression.

Conclusions: Overall, antidepressant use in Canadian children increased over the study period. Unsurprisingly, fluoxetine was the most recommended antidepressant for Canadian children. However, the observed increase in TCA use for a pediatric population is unexpected. The data source is descriptive and lacks detailed measures supporting comprehensive explanation of the findings, therefore, further research is required.
\end{abstract}

Keywords: antidepressants, pharmacoepidemiology, depression, pediatrics, tricyclics, prescription

\section{Introduction}

G UIDELINES FOR ANTIDEPRESSANT prescribing practices in pediatric populations have been reformulated multiple times since the 2000s. In the early 2000s, randomized controlled trials suggested that children under the age of 18 taking antidepressants exhibited increased risk of suicidal thoughts and behaviors compared with placebo groups (Hammad et al. 2006; Katz et al. 2008). In response, the Committee on Safety of Medicines in the United Kingdom issued a warning on paroxetine for children. Canada and the United States soon followed suit with Health Canada and the U.S. Food and Drug Administration's (FDA's) warnings on selective serotonin reuptake inhibitors (SSRIs), and selective norepinephrine reuptake inhibitors (SNRIs) in 2004.

However, evidence subsequently emerged indicating potential unintended consequences of the warnings-in addition to a subsequent drop in prescriptions, there was a coincident rise in the rate of completed suicides in children and adolescents observed in Canada, the United States, and the Netherlands (Gibbons et al. 2007; Katz et al. 2008).

Before the warning in 2004, the rate of prescriptions for children had been steadily increasing across North America (Olfson et al. 2002; Katz et al. 2008; Morkem et al. 2017). There is evidence that the decrease in prescriptions for children and adolescents in

\footnotetext{
${ }^{1}$ Department of Community Health Sciences, Cumming School of Medicine, University of Calgary, Calgary, Alberta, Canada.

${ }^{2}$ Department of Clinical Neurosciences, University of Calgary, Calgary, Alberta, Canada.

${ }^{3}$ Mathison Center for Research \& Education, Hotchkiss Brain Institute, University of Calgary, Calgary, Alberta, Canada.

${ }^{4}$ IQVIA, Ottawa, Ontario, Canada.

${ }^{5}$ Cuthbertson \& Fischer Chair in Pediatric Mental Health, University of Calgary, Calgary, Alberta, Canada.

Funding: This work was supported by the Cuthbertson \& Fischer Chair in Pediatric Mental Health and the University of Calgary, which supports Dr. Patten. David G. Stewart, Parco Chan, and Ali Tehrani are all employees of IQVIA Canada. The project received no financial support from IQVIA, but the data were provided to the investigators free of charge.
} 
response to the warning was brief, and numbers began to increase again soon after. Morkem's national time series analysis of primary care data for Canadian children suggests a decline in antidepressant prescriptions following the warning, but 5 years after, prescriptions had already returned to the previous rates (Morkem et al. 2017). Their analysis also shows an increase in prescription rates from 2009 to 2014 that exceeds the previously observed increase from 2000 to 2004.

Consistent with the Canadian data, from 2005 to 2012 antidepressant prescriptions for youth have risen in the United States, United Kingdom, Denmark, Germany, and The Netherlands (Bachmann et al. 2016). There have also been recent reports of increasing prescription rates in the United Kingdom and Australia (Karanges et al. 2014; John et al. 2016; Sarginson et al. 2017).

To date, there have been few Canadian studies reporting recent rates of prescriptions longitudinally after the warning. Lam and Morkem's analysis are two of the few national epidemiological studies, which describe prescribing trends longitudinally (Lam et al. 2013; Morkem et al. 2017). Both studies suggest an increase in prescriptions with Lam's focused on SSRIs prescribed by pediatricians. Conclusions indicate SSRIs are the most prescribed class of antidepressants, specifically fluoxetine, escitalopram, and sertraline (Lam et al. 2013; Morkem et al. 2017).

Fluoxetine has shown promise for providing a positive cost/benefit for treatment of depression in youth (Whittington et al. 2004). In addition to escitalopram, it is approved by the U.S. FDA for treatment of depression in pediatric patients (Eli Lilly 2017; Forest Pharmaceuticals 2017). Along with sertraline, fluvoxamine, and clomipramine, fluoxetine is also approved by the U.S. FDA for treatment of obsessive compulsive disorders (OCD) in pediatric populations ( Jazz Pharmaceuticals 2017; Mallinckrodt, Inc. 2017; Pfizer 2017; U.S Food \& Drug Administration 2018). However, in Canada, there are no antidepressant medications currently approved for youth under the age of 18 (HealthLink BC 2017).

The aim of this study is to investigate trends in antidepressant recommendations from 2012 to 2016 for Canadian children and adolescents to provide descriptive data and to help generate hypotheses for future research. The report updates and expands on Lam's previous findings on projected SSRI recommendations using a similar database. Additionally, our analysis will include data on classes of antidepressants, such as SNRIs, tricyclic antidepressants (TCAs), and other antidepressants. Also, we aim to describe trends of projected recommendations by patient diagnosis (indication), age group by diagnosis, class of antidepressant, and overall number and rate of recommendations.

\section{Methods}

\section{Canadian Disease and Therapeutic Index dataset}

Data were obtained from the Canadian Disease and Therapeutic Index (CDTI), which collects data on antidepressant recommendations by Canadian physicians and is maintained and updated by IQVIA. IQVIA maintains large curated health care data sources with advanced privacy protections, encompassing over 140,000 data sources worldwide with 600 million nonidentified patient records. Information on antidepressant recommendations over years 2012-2016 for children ages 1-18 is reported in the database. The CDTI data are one of a number of proprietary database products developed and maintained by IQVIA Canada.

The CDTI dataset is obtained through a survey administered to 652 Canadian office-based physicians stratified by physician specialty and geographic region. The pool of responding physicians is selected to be representative of all major office-based specialties, represented proportionally in a panel of participants recruited for the CDTI survey.

The CDTI physician panel is recruited by invitation and is updated annually. A minimum of $85 \%$ of reporting physicians in a current period are maintained from the previous physician sampling period. During the period of the study, repeat physician participation from quarter to quarter ranged from $89.6 \%$ to $100 \%$ (mean of $98.6 \%$ ). If a physician declines to participate in the survey, another physician of the same specialty and region is recruited to maintain the minimum physician sample size and panel representativeness. No monetary compensation was offered for participation. The estimates are weighted to produce statistically valid national estimates. However, as this is a proprietary data source, details of the weighting procedure are not available.

Physicians who take part in the survey report deidentified patient encounters detailing diagnostic indication and drug information for all patients seen during a 48-hour reporting period. Participating physicians complete this process four times each year. Demographic characteristics of physicians, such as age, sex, and duration of practice are not recorded by the IQVIA. Although, demographic characteristics of patients such as sex and exact age are recorded by the IQVIA, they were not disclosed in the provided version of the dataset. Since there is no personally identifiable information collected, informed consent is not required. Data on antidepressant recommendation class, subclass, indication, age category, and physician specialty are recorded along with other drug recommendations in the survey.

\section{Analyses}

Data were provided in the form of counts (estimated number of recommendations in the Canadian population). In the analyses we used counts, and calculated rates per 1000 person-years from January 2012 to December 2016 to observe changes in prescriptions recommended by physicians. We estimated rate of overall antidepressant recommendations using Statistic Canada's national midyear population estimates to confirm changes observed in the count data were not an artifact of population changes (Statistics Canada 2019).

All calculations and graphs were produced using Microsoft Excel 2017. The observed trends by year from 2012 to 2016 were the same for the count and rate of overall antidepressant recommendations. Therefore, with subsequent stratification, only projected counts representative of the population were used. All data were depicted graphically - in the absence of detailed information on the sampling procedure it is not possible to calculate confidence intervals or $p$-values. The projected prescription recommendations were stratified by: patient diagnosis (indication), age (categorized 1-6, 7-12, and 13-18), as well as subclass and specific medications.

\section{Results}

From 2012 to 2016 number and rate (1000 person-years) of projected antidepressant recommendations for children and adolescents increased as shown in Figure 1. Total number of projected antidepressant recommendations rose from 877,130 in 2012 to $1,193,517$ in 2016 , an increase of $36.07 \%$. Figure 1 demonstrates that the upward trend for rate and count do not appreciably differ, and therefore, the increasing trend observed is not merely attributed to a rise in the population of children and adolescents. For simplicity, subsequent figures and tables present only the count data. Table 1 describes the number of recommendations for each medication by year, including the top 3 indications associated with the recommendations. 
Projected Rate and Number of Antidepressant Recommendations in Children from 2012 to 2016

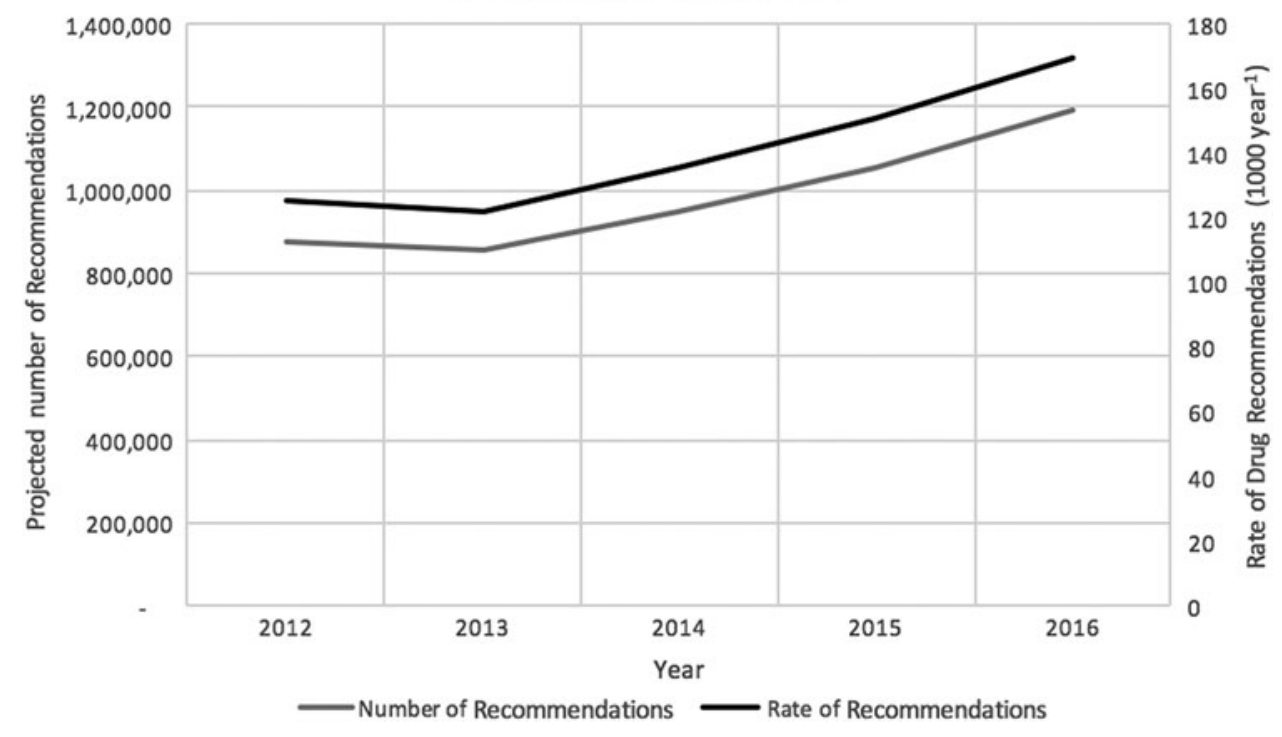

FIG. 1. Projected rate and number of antidepressant recommendations in children from 2012 to 2016.

SSRIs are estimated to be the most recommended subclass of antidepressant with fluoxetine as the most recommended in the group (Fig. 2). As shown in Table 1, out of all the SSRIs, fluoxetine also demonstrated a large increase throughout the years, with the 256,890 recommendations in 2012 jumping to 379,692 in 2016 , an increase of $47.80 \%$. The majority of fluoxetine recommendations were for children with anxiety disorders-in fact, the main indications associated with all estimated SSRI recommendations were depression and anxiety disorders (Table 1).

The least recommended SSRI throughout the years was fluvoxamine, dropping in recommendations from 52,230 to 16,362 , for a total decrease of $68.67 \%$ over the study period as shown by Table 1 and Figure 2. Although citalopram accounts for a significant portion of recommendations, it exhibited a decrease in recommendations by $7.68 \%$ from 2012 to 2016. Paroxetine also showed a decrease in recommendations by $14.04 \%$ over the study period (Table 1).

Estimated TCA recommendations have also increased from 2012 to 2016 with an overall increase of $114.36 \%$ (Fig. 3). Notably, the most common indications associated with TCA recommendations was "other" classification followed by Autism. In this dataset "other" includes any indication not listed. Amitriptyline accounted for the highest number of projected recommendations, and the most significant increase from 28,890 to 57,267 in 2012 and 2016, respectively (Table 1). Throughout the study period desipramine was never recommended. Doxepin, was also seldom used, as demonstrated in Table 1.

As found in Table 1, SNRIs and drugs classified as "other" exhibited an overall estimated increase during the study period. The most recommended SNRI being venlafaxine, and the most recommended in the "other" group being trazodone. Similar to previously described trends, depression and anxiety were the primary indications for SNRIs, however, in the "other" class in addition to these indications sleep disorders and bipolar disorders also accounted for a substantial portion of the recommendations.

The number of overall projected recommendations increased steadily by age group, where the 13-18 age group had 10 times the recommendations compared with the youngest $1-6$ group. Anxiety was the indication associated with the most estimated recommendations in all age groups other than ages 13-18, where it was surpassed by depression. Table 2 shows the number of projected recommendations per year for the top 5 indications, which include: attention-deficit/hyperactivity disorder (ADHD), anxiety disorders, bipolar disorders, depression, and OCD.

\section{Discussion}

Overall, projected antidepressant recommendations increased for Canadian children and adolescents during 2012 to 2016 according to a Canadian national data source. These findings are consistent with previous research on SSRIs during 2005 to 2009 , which also indicate increases in recommendations from pediatricians using the same database (Lam et al. 2013). Therefore, results suggest that the previously observed increase in antidepressant use following the warning continued during 2012-2016.

Our findings indicate that one of the major drivers of the observed rise in total recommendations, is the estimated increase in physicians recommending fluoxetine. Evidence from systematic reviews has suggested that fluoxetine may be the most effective antidepressant for youth (Ignaszewski and Waslick 2018). Fluoxetine has been approved in the United States by the FDA to treat anxiety disorders, OCD, and depression in children and adolescents (Geller et al. 2001; Birmaher et al. 2003). Also, evidence suggests that fluoxetine is effective for preventing relapse of depression in children and adolescents (Whittington et al. 2004; Ignaszewski and Waslick 2018). Whittington's systematic review of safety of SSRIs for depression in children concluded that fluoxetine was the only SSRI in which the benefits outweighed the risks (Whittington et al. 2004).

Over the study period, a projected number of recommendations of sertraline doubled. Although not approved for pediatric use in Canada, similar to fluoxetine, sertraline is approved by the FDA specifically for treatment of OCD in children. The number of sertraline recommendations are highest for anxiety and depression, 
Table 1. Overall Projected Recommendation by Drug and Top 3 Indications from 2012 to 2016

\begin{tabular}{|c|c|c|c|c|c|c|c|c|}
\hline Class & Drugs & Top 3 indications & Recommendations per indication & 2012 & 2013 & 2014 & 2015 & 2016 \\
\hline \multirow[t]{18}{*}{ SSRI } & \multirow[t]{3}{*}{ Citalopram } & Depression & 230,820 & \multirow[t]{3}{*}{126,610} & \multirow[t]{3}{*}{132,940} & \multirow[t]{3}{*}{67,872} & \multirow[t]{3}{*}{121,386} & \multirow[t]{3}{*}{116,882} \\
\hline & & Anxiety & 140,628 & & & & & \\
\hline & & Bipolar & 50,167 & & & & & \\
\hline & \multirow[t]{3}{*}{ Escitalopram } & Depression & 250,146 & \multirow[t]{3}{*}{99,200} & \multirow[t]{3}{*}{123,030} & \multirow[t]{3}{*}{116,470} & \multirow[t]{3}{*}{102,323} & \multirow{3}{*}{138,496} \\
\hline & & Anxiety & 183,035 & & & & & \\
\hline & & Bipolar & 97,560 & & & & & \\
\hline & \multirow{3}{*}{ Fluoxetine } & Anxiety & 631,031 & \multirow{3}{*}{256,890} & \multirow{3}{*}{253,910} & \multirow{3}{*}{388,029} & \multirow{3}{*}{345,423} & 379,692 \\
\hline & & Depression & 480,922 & & & & & \\
\hline & & ADHD & 133,972 & & & & & \\
\hline & Fluvoxamine & Anxiety & 63,647 & 52,230 & 26,440 & 21,790 & 13,832 & 16,362 \\
\hline & & OCD & 27,926 & & & & & \\
\hline & & Autism & 18,754 & & & & & \\
\hline & Paroxetine & Anxiety & 119,767 & 61,410 & 43,360 & 46,994 & 50,182 & 52,789 \\
\hline & & Depression & 103,848 & & & & & \\
\hline & & Personality disorder & 10,223 & & & & & \\
\hline & Sertraline & Anxiety & 227,688 & 87,240 & 99,210 & 64,447 & 164,058 & 175,336 \\
\hline & & Depression & 163,589 & & & & & \\
\hline & & OCD & 79,278 & & & & & \\
\hline SNRI & Desvenlafaxine & Depression & 53,975 & 13,600 & 10,310 & 21,594 & 16,341 & 13,588 \\
\hline & & Anxiety & 13,886 & & & & & \\
\hline & & Bipolar & 7572 & & & & & \\
\hline & Duloxetine & Anxiety & 27,154 & 6480 & 23,780 & 11,800 & 18,677 & 0 \\
\hline & & Depression & 16,130 & & & & & \\
\hline & & Bipolar & 14,143 & & & & & \\
\hline & Venlafaxine & Depression & 169,928 & 36,220 & 60,300 & 78,739 & 66,118 & 89,049 \\
\hline & & Anxiety & 122,016 & & & & & \\
\hline & & ADHD & 8155 & & & & & \\
\hline TCA & Amitriptyline & Other & 115,186 & 28,890 & 10,200 & 15,688 & 20,617 & 57,267 \\
\hline & & Depression & 9308 & & & & & \\
\hline & & Anxiety & 8168 & & & & & \\
\hline & Clomipramine & Autism & 20,692 & 6860 & 6810 & 3933 & 27,808 & 4684 \\
\hline & & OCD & 18,893 & & & & & \\
\hline & & Bipolar & 3523 & & & & & \\
\hline & Desipramine & NA & NA & 0 & 0 & 0 & 0 & 0 \\
\hline & Doxepin & Other $^{\mathrm{a}}$ & 1987 & 0 & 0 & 0 & 0 & 1987 \\
\hline & Imipramine & Other $^{a}$ & 15,440 & 0 & 5670 & 13,104 & 7992 & - \\
\hline & & Anxiety & 5663 & & & & & \\
\hline & & Sleep disorder & 2999 & & & & & \\
\hline & Nortriptyline & Other $^{\mathrm{a}}$ & 16,207 & 5540 & 0 & 6956 & 0 & 20,749 \\
\hline & Trimipramine & Other $^{\mathrm{a}}$ & 16,207 & 0 & 0 & 0 & 0 & 16,207 \\
\hline Other & Bupropion & Depression & 68,742 & 29,600 & 18,150 & 30,926 & 18,947 & 39,060 \\
\hline & & Bipolar & 23,229 & & & & & \\
\hline & & Anxiety & 22,805 & & & & & \\
\hline & Mirtazapine & Anxiety & 23,507 & 3370 & 3500 & 11,800 & 8851 & 26,672 \\
\hline & & Depression & 20,719 & & & & & \\
\hline & & Autism & 3933 & & & & & \\
\hline & Moclobemide & NA & NA & 0 & 0 & 0 & 0 & 0 \\
\hline & Trazodone & Sleep disorder & 105,304 & 62,990 & 35,990 & 45,656 & 63,753 & 36,595 \\
\hline & & Depression & 38,568 & & & & & \\
\hline & & Autism & 36,698 & & & & & \\
\hline & Vortioxetine & Bipolar & 8104 & 0 & 0 & 0 & 6013 & 8104 \\
\hline & & Depression & 6013 & & & & & \\
\hline
\end{tabular}

${ }^{a}$ Recommendations for any indication other than the following: ADHD, adjustment disorder, anxiety disorder, autism, bipolar disorder, conduct disorder, depression, developmental delay, drug addiction, eating disorder, OCD, personality disorder, psychotic disorder, sleep disorder, somatoform disorders, and Tic disorder.

ADHD, attention-deficit/hyperactivity disorder; OCD, obsessive/compulsive disorder; SNRI, selective norepinephrine reuptake inhibitor; SSRI, selective serotonin reuptake inhibitor; TCA, tricyclic antidepressant.

followed by OCD (Table 1), but as expected, sertraline makes up the highest percentage of recommendations associated with OCD in the dataset. Also, anxiety is an important manifestation of OCD, so some of the increase may represent treatment of OCD. From 2012 to 2016 sertraline accounted for $31.89 \%$ of all recommen- dations associated with OCD, but only $14.49 \%$ and $10.02 \%$ of those for anxiety and depression, respectively. Throughout the study period, proportion of estimated sertraline recommendations associated with anxiety and depression more than doubled, while proportion associated with OCD more than halved. There is evidence 


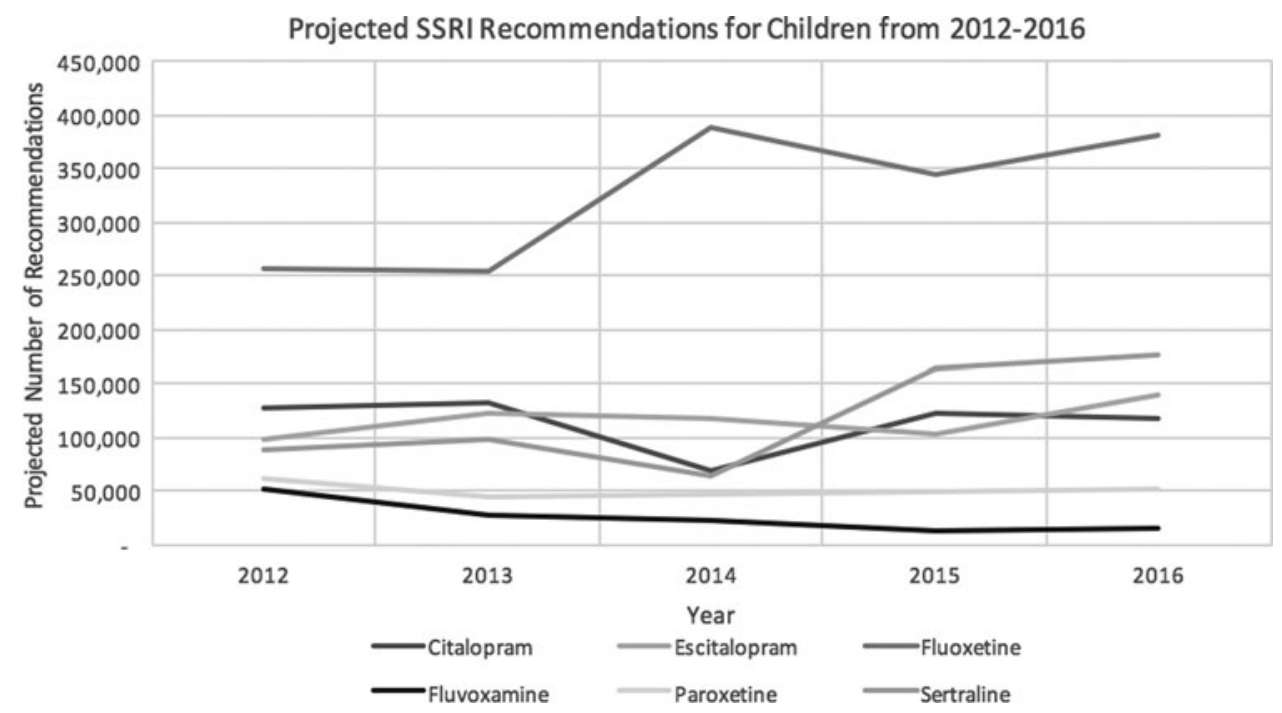

FIG. 2. Projected SSRI recommendations for children from 2012 to 2016 . SSRI, selective serotonin reuptake inhibitor.

suggesting that benefits of sertraline treatment may outweigh the risk of suicide in children for treatment of OCD, anxiety, and major depressive disorder (Strawn et al. 2015; Varigonda et al. 2015; Öst et al. 2016). Therefore, in the context of its FDA approval, it is expected that physicians would increasingly recommend sertraline. To determine the reason for trends relating to the associated indication, further research is required.

As expected, the projected number of overall TCA recommendations are lower than that of overall SSRIs. Concerns related to sudden cardiac death in children extend to all TCAs (Varley 2001; Amitai and Frischer 2006). However, recommendations of TCAs doubled over this time interval, with an increase particularly in the latter years of the study period. These findings are unexpected, since there is scarce evidence to suggest TCAs are effective in treating children and adolescents.

Generally, extant research suggests that TCAs are unfavorable for treatment in pediatric populations due to the potential for tox- icity from overdose, especially negative cardiac effects (Emslie and Judge 2000; Hazell and Mirzaie 2013). Since the data recorded estimated recommendations rather than quantities, it is possible that physicians are providing smaller prescriptions more frequently, leading to an apparent increase in recommendations. Another possibility is that TCAs are increasingly being recommended for nonpsychiatric indications. Evidence indicates TCAs, such as imipramine, amitriptyline, and desipramine, are an effective treatment for nocturnal enuresis (Caldwell et al. 2016). Additionally, a recent Scottish national data source exhibited similar findings, where use of amitriptyline increased from 2006 to 2017, largely from use for other nonpsychiatric indications (NHS 2017).

Amitriptyline had the highest number of recommendations and exhibited the most significant increase out of all TCAs over the study period. Evidence suggests that amitriptyline may be effective for the treatment of chronic and neuropathic pain in children (Goldenberg et al. 1986; Hershey et al. 2000). Since the primary

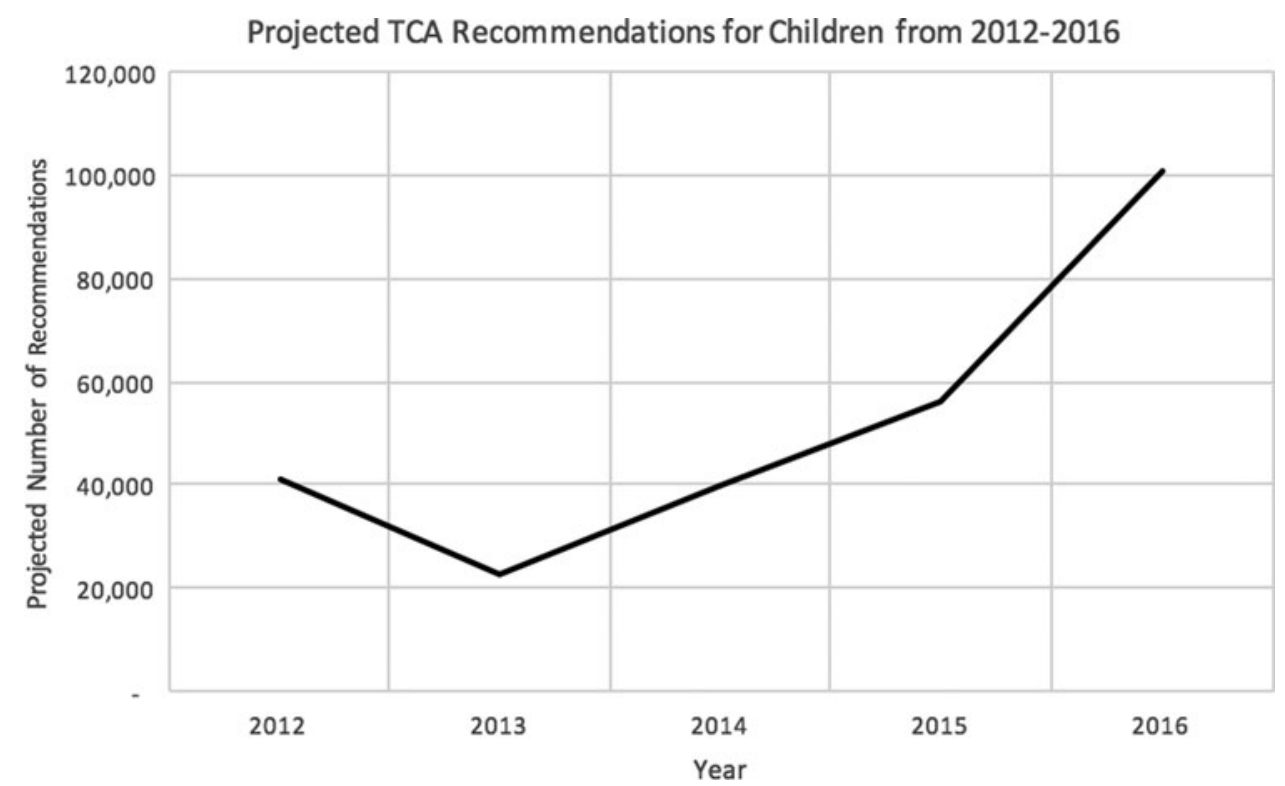

FIG. 3. Projected TCA recommendations for children from 2012 to 2016 . TCA, tricyclic antidepressant. 
Table 2. Overall Projected Recommendations FOR THE TOP 5 INDICATIONS

\begin{tabular}{llllll}
\hline & 2012 & 2013 & 2014 & 2015 & 2016 \\
\hline ADHD & 37,080 & 20,380 & 48,643 & 58,415 & 50,854 \\
Anxiety disorder & 248,270 & 313,830 & 289,207 & 359,178 & 402,003 \\
Bipolar disorder & 88,410 & 78,050 & 60,686 & 44,834 & 59,835 \\
Depression & 236,000 & 242,960 & 383,196 & 332,364 & 438,236 \\
OCD & 49,370 & 86,780 & 15,826 & 49,883 & 46,737 \\
\hline
\end{tabular}

ADHD, attention-deficit/hyperactivity disorder; OCD, obsessive compulsive disorder.

indication for amitriptyline recommendations was classified by the IQVIA as "other," it is possible that indications related to pain management may be the source of such findings. In fact, as demonstrated by Table 1, the projected number of recommendations for depression and anxiety are minimal compared with the "other" indication. More concerning, is the alternate possibility that physicians prescribing the TCAs are unaware of the troubling side effects in children. However, additional research using a more detailed data sources is required to assess these hypotheses.

Another unexpected finding is the high number of projected recommendations attributed to bipolar disorder. Bipolar is listed as a top 3 indication for estimated recommendations of citalopram, escitalopram, desvenlafaxine, duloxetine, bupropion, and vortioxetine (Table 1). Antidepressant use for bipolar can be contraindicated due to concerns of inducing a manic/hypomanic episode (Pacchiarotti et al. 2013). However, The International Society for Bipolar Disorders created a task force to determine a consensus for recommendations for use of antidepressants in bipolar disorders (Pacchiarotti et al. 2013). The consensus was that SSRIs and bupropion are least likely to induce mania compared with other antidepressants (Pacchiarotti et al. 2013). These suggestions are concordant with the findings presented in Table 1, where SSRIs and bupropion are the antidepressants most attributed to bipolar.

Therefore, a conjecture can be made that these estimated recommendations might be attributable to bipolar depression, where in conjunction with an antimanic agent, antidepressants can be an effective treatment (Pacchiarotti et al. 2013; Gijsman et al. 2004). Also, these antidepressants may be prescribed briefly to address a bipolar depressive episode, and discontinued shortly after. As previously mentioned, information regarding quantities of the drugs being recommended, and duration of prescription is not provided by the CDTI database. Therefore, further research to confirm such hypotheses is necessary to fully understand the trends and the associated concerns.

More enigmatic, is the estimated recommendations of fluoxetine and venlafaxine attributed to ADHD (Table 1). Little evidence exists to support the use of antidepressants for ADHD alone (atomoxetine was not classified as an antidepressant in the analysis); however, it is possible that recommendations associated with ADHD were used to address ADHD with comorbid depression or anxiety. Previous research suggests that children with these psychiatric comorbidities may benefit from combined therapies of atomoxetine and fluoxetine (Kratochvil et al. 2005) or use of Venlafaxine (Motavalli Mukaddes and Abali 2004; Ghanizadeh et al. 2013). Due to the lack of detail in the database, it is difficult to justify such anomalies and further research is required to investigate potentially harmful prescribing practices.

There are various hypotheses that may be formed to explain the overall increase in estimated recommendations exhibited by the results. A reduction of stigma, improvement of health services, and increased access to care, would all serve as possible explanations for the increase in recommendations over time. Similarly, increasing frequencies of various underlying indications such as depression and anxiety may explain the increase. Alternatively, over-recommending antidepressants would result in the same trend, and could arise as a result of diminishing availability of nonpharmacological treatments. Further research using a more detailed data source is required to adequately support any given hypothesis generated from our results.

Although the CDTI data from IQVIA provide valuable information on physician's recommendation practices in Canada, there are also several associated limitations. Due to sampling techniques, results may be influenced by the Hawthorne effect (McCarney et al. 2007). Since physicians participate voluntarily, they are not blinded. Consequently, they may change their prescribing behaviors during the reporting period, for example, to adhere more closely to guidelines. Relatedly, since recommendations are self-reported, it is possible that physicians could have untruthfully reported their prescribing behaviors.

These data represent recommendations by physicians and it is not known if patients subsequently fill their prescriptions. Notably, there is no additional description provided for the category of "other" for indications in the database. Consequently, the category could describe other psychiatric diagnoses not listed, or other nonpsychiatric indications. With such limitations it is not possible to make conclusions regarding the underlying reasons for the rise in tricyclic recommendations.

The dataset also lacks information on the duration of prescriptions that are dispensed, and if patients switch medications, or discontinue use. The datasets are provided by IQVIA, which is a private enterprise that projects the data to be representative of the Canadian population. Detailed information regarding sampling and projection techniques and raw data are not provided to researchers, so unexpected trends are difficult to further analyze and explain and the possible appearance of trends due to random sampling variability cannot be quantified statistically. Also, specific information on attrition rates of physicians between years, and response number by year is not provided by the IQVIA. In this context, our results should be primarily interpreted as hypothesis generating for further research using a more detailed data source to further analyze the unexpected results obtained in our analysis.

\section{Conclusions}

Overall, there has been an increasing trend in the IQVIA's projected antidepressant recommendations for the Canadian pediatric populations, ages 1-18, during 2012-2016. Fluoxetine was the most recommended antidepressant. Findings which indicate that use of TCAs may be increasing are surprising and provide fertile grounds for further research using more detailed data sources.

\section{Clinical Significance}

Our study is one of the few recent epidemiological reports of pediatric prescribing trends in Canada. With future research using a more detailed dataset, our findings contribute to the understanding of preferred physician practices relating to antidepressant prescription trends, which may help to develop best practice guidelines. In particular, the clinical appropriateness of the increased prescribing and increasing use of TCAs requires further investigation. Findings also help guide further research investigating the rise in antidepressant prescriptions for pediatric populations in Canada. 


\section{Disclosures}

Aysha Lukmanji: No conflicts of interest to declare. Tamara Pringsheim: Receives funding from the Owerko Center of the Alberta Children's Hospital Research Institute, no conflicts of interest to declare. Andrew G. Bulloch: No conflicts of interest to declare. David G. Stewart: Is an employee of IQVIA. Parco Chan: Is an employee of IQVIA. Ali Tehrani: Is an employee of IQVIA. Scott B. Patten: Is supported by the Cuthbertson \& Fischer Chair in Pediatric Mental Health and the University of Calgary. No conflicts of interest to declare.

\section{References}

Amitai Y, Frischer H: Excess fatality from desipramine in children and adolescents. J Am Acad Child Adolesc Psychiatry 45:54-60, 2006.

Bachmann CJ, Aagaard L, Burcu M, Glaeske G, Kalverdijk LJ, Petersen I, Schuiling-Veninga CC, Wijlaars L, Zito JM, Hoffmann F: Trends and patterns of antidepressant use in children and adolescents from five western countries, 2005-2012. Eur Neuropsychopharmacol 26:411-419, 2016.

Birmaher B, Axelson DA, Monk K, Kalas C, Clark DB, Ehmann M, Bridge J, Heo J, Brent DA: Fluoxetine for the treatment of childhood anxiety disorders. J Am Acad Child Adolesc Psychiatry 42: 415-423, 2003.

Caldwell PH, Sureshkumar P, Wong WC: Tricyclic and related drugs for nocturnal enuresis in children. Cochrane Database Syst RevCD002117, 2016. DOI: 10.1002/14651858.CD002117.pub2.

Eli Lilly: PROZAC (fluoxetine) [package insert]. Food and Drug Administration, 2017.

Emslie G, Judge R: Tricyclic antidepressants and selective serotonin reuptake inhibitors: Use during pregnancy, in children/adolescents and in the elderly. Acta Psychiatr Scand Suppl 403:26-34, 2000.

Forest Pharmaceuticals: LEXAPRO (escitalopram) [package insert]. Food and Drug Administration, 2017.

Geller DA, Hoog SL, Heiligenstein JH, Ricardi RK, Tamura R, Kluszynski S, Jacobson JG: Fluoxetine treatment for obsessivecompulsive disorder in children and adolescents: A placebocontrolled clinical trial. J Am Acad Child Adolesc Psychiatry 40: 773-779, 2001.

Ghanizadeh A, D Freeman R, Berk M: Efficacy and adverse effects of venlafaxine in children and adolescents with ADHD: A systematic review of non-controlled and controlled trials. Rev Recent Clin Trials 8:2-8, 2013.

Gibbons RD, Brown CH, Hur K, Marcus SM, Bhaumik DK, Erkens JA, Herings RM, Mann JJ: Early evidence on the effects of regulators' suicidality warnings on SSRI prescriptions and suicide in children and adolescents. Am J Psychiatry 164:1356-1363, 2007.

Gijsman HJ, Geddes JR, Rendell JM, Nolen WA, Goodwin GM: Antidepressants for bipolar depression: A systematic review of randomized, controlled trials. Am J Psychiatry 161:1537-1547, 2004.

Goldenberg DL, Felson DT, Dinerman H: A randomized, controlled trial of amitriptyline and naproxen in the treatment of patients with fibromyalgia. Arthritis Rheum 29:1371-1377, 1986.

Hammad TA, Laughren T, Racoosin J: Suicidality in pediatric patients treated with antidepressant drugs. Arch Gen Psychiatry 63: 332-339, 2006.

Hazell P, Mirzaie M: Tricyclic drugs for depression in children and adolescents. Cochrane Database Syst Rev CD002317, 2013. DOI: 10.1002/14651858.CD002317.pub2.

HealthLink BC: Health Canada and FDA Advisories for Antidepressants. December 7, 2017.
Hershey AD, Powers SW, Bentti AL, DeGrauw TJ: Effectiveness of amitriptyline in the prophylactic management of childhood headaches. Headache 40:539-549, 2000.

Ignaszewski MJ, Waslick B: Update on randomized placebocontrolled trials in the past decade for treatment of major depressive disorder in child and adolescent patients: A systematic review. J Child Adolesc Psychopharmacol 28:668-675, 2018.

Jazz Pharmaceuticals: LUVOX (Fluvoxamine maleate) [package insert]. Food and Drug Administration, 2017.

John A, Marchant A, Fone D, McGregor J, Dennis M, Tan J, Lloyd K: Recent trends in primary-care antidepressant prescribing to children and young people: An e-cohort study. Psychol Med 46:3315-3327, 2016.

Karanges EA, Stephenson CP, McGregor IS: Longitudinal trends in the dispensing of psychotropic medications in Australia from 20092012: Focus on children, adolescents and prescriber specialty. Aust N Z J Psychiatry 48:917-931, 2014.

Katz LY, Kozyrskyj AL, Prior HJ, Enns MW, Cox BJ, Sareen J: Effect of regulatory warnings on antidepressant prescription rates, use of health services and outcomes among children, adolescents and young adults. CMAJ 178:1005-1011, 2008.

Kratochvil CJ, Newcorn JH, Arnold LE, Duesenberg D, Emslie GJ, Quintana H, Sarkis EH, Wagner KD, Gao H, Michelson D: Atomoxetine alone or combined with fluoxetine for treating ADHD with comorbid depressive or anxiety symptoms. J Am Acad Child Adolesc Psychiatry 44:915-924, 2005.

Lam D, Gorman DA, Patten S, Pringsheim T: The pharmacoepidemiology of selective serotonin reuptake inhibitors for children and adolescents in Canada from 2005 to 2009: A database analysis. Paediatr Drugs 15:319-327, 2013.

Mallinckrodt, Inc.: ANAFRANIL (clomipramine) [package insert]. Food and Drug Administration, 2017.

McCarney R, Warner J, Iliffe S, Van Haselen R, Griffin M, Fisher P: The Hawthorne effect: A randomised, controlled trial. BMC Med Res Methodol 7:30, 2007.

Morkem R, Williamson T, Patten S, Queenan JA, Wong ST, Manca $\mathrm{D}$, Barber D: Trends in antidepressant prescribing to children and adolescents in Canadian primary care: A time-series analysis. Pharmacoepidemiol Drug Saf 26:1093-1099, 2017.

Motavalli Mukaddes N, Abali O: Venlafaxine in children and adolescents with attention deficit hyperactivity disorder. Psychiatry Clin Neurosci 58:92-95, 2004.

NHS: National Services Scotland. Information services division publication report. Medicines used in mental health, years 2006/07 to 2016/17. 2017. Available at: https://www.isdscotland.org/HealthTopics/Prescribing-and-Medicines/Publications/2017-10-10/2017-1010-PrescribingMentalHealth-Report.pdf Accessed December 20, 2019.

Olfson M, Marcus SC, Weissman MM, Jensen PS: National trends in the use of psychotropic medications by children. J Am Acad Child Adolesc Psychiatry 41:514-521, 2002.

Öst L-G, Riise EN, Wergeland GJ, Hansen B, Kvale G: Cognitive behavioral and pharmacological treatments of OCD in children: A systematic review and meta-analysis. J Anxiety Disord 43:58-69, 2016.

Pacchiarotti I, Bond DJ, Baldessarini RJ, Nolen WA, Grunze H, Licht RW, Post RM, Berk M, Goodwin GM, Sachs GS: The International Society for Bipolar Disorders (ISBD) task force report on antidepressant use in bipolar disorders. Am J Psychiatry 170:1249-1262, 2013.

Pfizer: ZOLOFT (sertraline) [package insert]. Food and Drug Administration, 2017.

Sarginson J, Webb RT, Stocks SJ, Esmail A, Garg S, Ashcroft DM: Temporal trends in antidepressant prescribing to children in UK primary care, 2000-2015. J Affect Disord 210:312-318, 2017. 
Statistics Canada: Canada's population estimates: Age and sex, July 1, 2019. 2019. Available at: https://www 150 .statcan.gc.ca/n1/dailyquotidien/190930/dq190930a-eng.htm Accessed December 20, 2019.

Strawn JR, Welge JA, Wehry AM, Keeshin B, Rynn MA: Efficacy and tolerability of antidepressants in pediatric anxiety disorders: A systematic review and meta-analysis. Depress Anxiety 32:149-157, 2015.

U.S. Food and Drug Administration: Suicidality in Children and Adolescents Being Treated With Antidepressant Medications. 2018.

Varigonda AL, Jakubovski E, Taylor MJ, Freemantle N, Coughlin C, Bloch MH: Systematic review and meta-analysis: Early treatment responses of selective serotonin reuptake inhibitors in pediatric major depressive disorder. J Am Acad Child Adolesc Psychiatry 54: 557-564, 2015.

Varley CK: Sudden death related to selected tricyclic antidepressants in children. Paediatr Drugs 3:613-627, 2001.
Whittington CJ, Kendall T, Fonagy P, Cottrell D, Cotgrove A, Boddington E: Selective serotonin reuptake inhibitors in childhood depression: Systematic review of published versus unpublished data. Lancet 363:1341-1345, 2004.

\author{
Address correspondence to: \\ Scott B. Patten, MD, PhD \\ Department of Community Health Sciences \\ Cumming School of Medicine \\ University of Calgary \\ TRW Building, Room 4D66 \\ 3280 Hospital Drive NW \\ Calgary, AB T2N 4N1 \\ Canada
}

E-mail: patten@ucalgary.ca 